\title{
Soroprevalência de leptospirose em cavalos de corrida: um estudo observacional
}

\section{descritivo}

\author{
Leptospirosis seroprevalence in racehorses: a descriptive observational study \\ Seroprevalencia de leptospirosis en caballos de carrera: un estudio observacional descriptivo
}

Recebido: 16/06/2021 | Revisado: 24/06/2021 | Aceito: 29/06/2021 | Publicado: 12/07/2021

\author{
Vitória Müller \\ ORCID: https://orcid.org/0000-0002-4585-6772 \\ Universidade Federal de Pelotas, Brasil \\ E-mail: mullervitoria@hotmail.com \\ Bruna dos Santos Suñé Moraes \\ ORCID: https://orcid.org/0000-0002-4823-2906 \\ Universidade Federal de Pelotas, Brasil \\ E-mail: brunasune@ hotmail.com \\ Rafaela Pinto de Souza \\ ORCID: https://orcid.org/0000-0003-0662-6728 \\ Universidade Federal de Pelotas, Brasil \\ E-mail: rafaelapsvet@gmail.com \\ Mariana Andrade Mousquer \\ ORCID: https://orcid.org/0000-0001-8253-3713 \\ Universidade Federal de Pelotas, Brasil \\ E-mail: mmousquer.vet@gmail.com \\ Caroline Dewes \\ ORCID: https://orcid.org/0000-0002-7404-0975 \\ Universidade Federal de Pelotas, Brasil \\ E-mail: caroldewesvet@hotmail.com \\ Éverton Fagonde da Silva \\ ORCID: https://orcid.org/0000-0002-4226-7235 \\ Universidade Federal de Pelotas, Brasil \\ E-mail: fagondee@gmail.com \\ Carlos Eduardo Wayne Nogueira \\ ORCID: https://orcid.org/0000-0002-8555-7953 \\ Universidade Federal de Pelotas, Brasil \\ E-mail: cewn@gmail.com
}

\begin{abstract}
Resumo
Este estudo teve como objetivo determinar a soroprevalência de leptospirose em cavalos de corrida hospedados no Jockey Club de Pelotas (JCP) e avaliar os fatores de risco relacionados a essa doença. Foram incluídos 84 equinos da Raça Puro Sangue Inglês. Os animais foram submetidos à avaliação clínica geral e a colheita de sangue para obtenção de amostras de soro. Para detecção de anticorpos anti-leptospiras foi realizado o teste de soroaglutinação microscópica (MAT) para os seguintes sorovares de L. interrogans: Australis, Autumnalis, Batavie, Bratislava, Copenhageni, Grippothyphosa, Hardjoprajitno, Icterohaemorrhagiae, Pomona, Pyrogenes e Patoc, sendo considerado reagente quando a aglutinação foi $\geq 1: 100$. Posteriormente, um único entrevistador aplicou um questionário epidemiológico aos responsáveis pelos equinos para determinar a presença de fatores de risco para leptospirose no local. Dentre os 84 equinos, apenas nove $(10,7 \%)$ apresentaram titulação para L. interrogans. As titulações variaram entre 1:100 e 1:400, sendo que três equinos apresentaram titulação para mais de um sorovar. Os sorovares identificados neste estudo foram Canicola (33,3\%), Grippotyphose (33,3\%), Icterohaemorrhagiae (22,2\%), Australis (22,2\%), Pyrogenes (11,1\%), Bratislava (11,1\%), Copenhageni $(11,1 \%)$, Autumnalis $(11,1 \%)$ e Hardjoprajitno $(11,1 \%)$. Não houve associação entre os resultados do teste sorológico e os dados obtidos através do questionário epidemiológico ( $\mathrm{p}>0,05)$. Através deste estudo verificou-se que a soroprevalência para L. interrogans em equinos do JCP é baixa. Estudos epidemiológicos em outros hipódromos são necessários para determinar fatores de risco que estejam associados à infecção por $L$. interrogans em cavalos de corrida.
\end{abstract}

Palavras-chave: L. interrogans; Equino; Fatores de risco; Zoonose.

\begin{abstract}
This study aimed to determine the seroprevalence of leptospirosis in racehorses hosted at the Jockey Club of Pelotas (JCP) and the risk factors related to this disease. Eighty-four thoroughbred horses were included in this study. The animals were submitted to physical exams and were bled to obtain serum samples. Microscopic agglutination test (MAT) was performed for the following L. interrogans serovars: Australis, Autumnalis, Batavie, Bratislava,
\end{abstract}


Copenhageni, Grippothyphosa, Hardjoprajitno, Icterohaemorrhagiae, Pomona, Pyrogenes and Patoc, being considered reagent when agglutination was $\geq 1: 100$. Subsequently, a single interviewer applied an epidemiological questionnaire to those responsible for the horses to determine the presence of risk factors for leptospirosis at the JCP. Among the 84 horses, only nine $(10.7 \%)$ had titers for L. interrogans. Titers ranged between 1:100 and 1:400, and three horses had titers for more than one serovar. The serovars identified in this study were Canicola (33.3\%), Grippotyphose (33.3\%), Icterohaemorrhagiae (22.2\%), Australis (22.2\%), Pyrogenes (11.1\%), Bratislava (11,1\%), Copenhageni $(11.1 \%)$, Autumnalis $(11.1 \%)$ and Hardjoprajitno $(11.1 \%)$. There was no association between the results of the serological test and the data obtained through the epidemiological questionnaire $(\mathrm{p}>0.05)$. This study demonstrated that the seroprevalence for L. interrogans in horses hosted at the JCP is low. Epidemiological studies in other racecourses to determine risk factors that are associated with L. interrogans infection in racehorses.

Keywords: L. interrogans; Equine; Risk factors; Zoonoses.

\section{Resumen}

Este estudio tuvo como objetivo determinar la seroprevalencia de leptospirosis en caballos de carrera alojados en el Jockey Club de Pelotas (JCP) y los factores de riesgo relacionados con esta enfermedad. Se incluyeron 84 caballos Pura Sangre Inglesa. Los animales fueron sometidos a evaluación clínica y extracción de sangre para obtener muestras de suero. Para la detección de anticuerpos anti-leptospiras se realizó la prueba de aglutinación microscópica (MAT) para los siguientes serovares de L. interrogans: Australis, Autumnalis, Batavie, Bratislava, Copenhageni, Grippothyphosa, Hardjoprajitno, Icterohaemorrhagiae, Pomona, Pyrogenes y Patoc, siendo considerados reactivos cuando la aglutinación fue $\geq 1: 100$. Posteriormente, un entrevistador aplicó un cuestionario epidemiológico a los responsables de los caballos para determinar la presencia de factores de riesgo de leptospirosis en el sitio. Entre los 84 caballos, solo nueve $(10,7 \%)$ tenían un título para L. interrogans. Los títulos variaron entre 1:100 y 1:400, con tres caballos titulados a más de un serovar. Las serovares identificadas fueron Canicola (33,3\%), Grippotyphose (33,3\%), Icterohaemorrhagiae (22,2\%), Australis (22,2\%), Pyrogenes (11,1\%), Bratislava (11,1\%), Copenhageni (11,1\%), Autumnalis $(11,1 \%)$ y Hardjoprajitno $(11,1 \%)$. No hubo asociación entre los resultados de la prueba serológica y los datos obtenidos através del cuestionario epidemiológico ( $p>0,05)$. Através de este estudio se encontró que la seroprevalencia de L. interrogans en caballos del JCP es baja. Se necesitan estudios epidemiológicos en otros hipódromos para determinar los factores de riesgo asociados con la infección por L. interrogans en caballos de carrera.

Palabras clave: L. interrogans; Equino; Factores de riesgo; Zoonosis.

\section{Introdução}

A leptospirose é uma zoonose de distribuição cosmopolita causada por espécies patogênicas da bactéria do gênero Leptospira (Sellon \& Long, 2007). Além de representar um risco para a saúde pública, a leptospirose cursa com perdas econômicas na equideocultura devido à ocorrência de abortos, queda do desempenho atlético e custos com o tratamento (Ebani et al., 2012; Chang, 2021). A espécie L. interrogans é a principal responsável pela infecção de equinos (Verma et al., 2013). A transmissão da leptospirose ocorre através do contato direto com a urina de animais contaminados, fontes de água, solo e alimentos contaminados, assim como através do contato com fetos abortados, fômites e do contato sexual (Faine, 1982; Levett, 2001; Divers et al., 2019).

Os roedores desempenham um papel fundamental no ciclo da leptospirose, pois se tornam portadores assintomáticos que passam a portar a Leptospira spp. no interior dos túbulos renais e a excretá-la na urina, contaminando o ambiente (Miraglia et al., 2013). Além dos roedores, outros animais podem atuar como fontes de contaminação através da leptospiúria como cães, gatos, preás e gambás, além do próprio equino (Thomasian, 2005). Outros fatores que contribuem para a disseminação do agente são a geografia plana e elevados índices pluviométricos, os quais dificultam o escoamento da água e contribuem para o aumento da incidência desta enfermidade (Verma et al., 2013).

O Jockey Club de Pelotas (JCP), também chamado de Hipódromo da Tablada, está situado na área urbana do município de Pelotas-RS, onde permanecem albergados equinos da raça Puro Sangue Inglês (PSI), os quais participam eventos turfísticos no local e em outras cidades. A hospedagem de equinos neste local gera a necessidade de armazenamento de alimentos que atraem roedores e outros animais presentes na área urbana, e que podem contribuir para o aumento da incidência de leptospirose na cidade de Pelotas. Além disso, o JCP apresenta uma geografia plana, o que dificulta o escoamento da água nos períodos de aumento dos índices pluviométricos. A realização de um estudo epidemiológico sobre leptospirose neste local 
pode auxiliar no desenvolvimento de estratégias para o combate desta zoonose. Portanto, este estudo teve como objetivos: (1) determinar a soroprevalência de leptospirose equina em cavalos de corrida hospedados no JCP; (2) avaliar os fatores de risco relacionados a essa doença no local estudado.

\section{Metodologia}

Um estudo observacional descritivo foi realizado no ano de 2019, no Jockey Club de Pelotas, localizado na cidade de

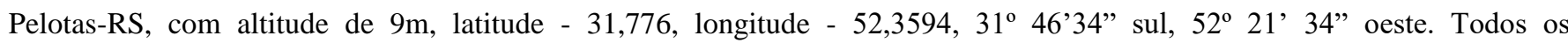
procedimentos realizados foram previamente aprovados pelo Comitê de Ética em Experimentação Animal (CEEA) da Universidade Federal de Pelotas sob o número de protocolo: 42884-2019.

\subsection{Animais e coletas}

Foram incluídos neste estudo todos os equinos hospedados no Jockey Club de Pelotas na data estipulada para realização das coletas, compreendendo um total de 84 equinos da Raça Puro Sangue Inglês. Todos os animais foram submetidos à avaliação clínica geral e, posteriormente, à coleta de amostras de sangue através da venopunção da jugular externa utilizando tubos sem anticoagulante e agulhas vacutainer $25 \times 7$. As amostras foram mantidas em repouso e, após a retração do coágulo, foram centrifugadas a $600 \mathrm{X}$ g por 10 minutos para obtenção de soro e armazenamento do mesmo a -20 ${ }^{\circ} \mathrm{C}$ até posterior análise.

\subsection{Teste de soroaglutinação microscópica (MAT)}

Todas as amostras de soro foram descongeladas em temperatura ambiente e testadas em um painel de antígenos formados por 10 sorovares de leptospiras vivas causadoras de leptospirose no Brasil: Australis, Autumnalis, Batavie, Bratislava, Copenhageni, Grippothyphosa, Hardjoprajitno, Icterohaemorrhagiae, Pomona e Pyrogenes, os quais foram cedidos pela Fundação Oswaldo Cruz (FIOCRUZ - RJ), acrescido do sorovar saprófita Patoc. O MAT foi realizado conforme descrito anteriormente por Dewes et al. (2020) e de acordo com as recomendações da Organização Mundial de Saúde (WHO, 2003). As amostras foram submetidas à pesquisa de aglutininas anti-leptospiras em diluições crescentes de 1:100 até 1:3200, e o teste foi considerado reagente quando houve aglutinação $\geq 1: 100$ (Santa Rosa, 1970).

\subsection{Questionário epidemiológico}

Após as coletas de sangue, um único entrevistador aplicou um questionário epidemiológico a todos os responsáveis pelos equinos hospedados no JCP a fim de obter informações referentes ao indivíduo, manejo e controle sanitário destes animais. Foram incluídas no questionário as variáveis: idade, gênero (fêmea, macho castrado e macho inteiro), número de páreos disputados no JCP nos anos de 2018/2019, número de páreos disputados em outras cidades nos anos de 2018/2019, tipo de cama (maravalha, casca de arroz, maravalha e casca de arroz), dieta (consumo de alfafa, ração comercial, aveia, milho e sal mineral), forma de armazenamento dos grãos (saco, tonel, baú, saco e tonel), vacinação prévia contra leptospirose, presença de animais de estimação no conjunto de cocheiras, acesso dos animais de estimação à sala da ração, histórico de roedores no local, presença de pessoas residindo no conjunto de cocheiras.

\subsection{Análises estatísticas}

Foi realizada a estatística descritiva e as variáveis quantitativas foram descritas como Média \pm EPM, enquanto as variáveis qualitativas foram descritas como frequência e percentual. Para analisar variáveis quantitativas entre animais com e sem titulação para leptospirose no MAT foi realizado o Teste-t independente, enquanto as variáveis qualitativas foram 
comparadas pelo Teste Exato de Fisher. As análises estatísticas foram realizadas através do programa IBM SPSS Statistics 20 e foi considerada diferença estatística quando $\mathrm{p} \leq 0,05$.

\section{Resultados}

Dos 84 equinos incluídos neste estudo, nove $(10,7 \%)$ apresentaram titulação para L. interrogans. As titulações variaram entre 1:100 e 1:400, sendo que três equinos apresentaram titulação para mais de um sorotipo. Os sorovares identificados neste estudo foram Canicola (3/84; 33,3 \%), Grippotyphosa (3/84; 33,3\%), Icterohaemorrhagiae (2/84; 22,2 \%), Australis $(2 / 84 ; 22,2 \%)$, Pyrogenes $(1 / 84 ; 11,1 \%)$, Bratislava (1/84; 11,1\%), Copenhageni $(1 / 84 ; 11,1 \%)$, Autumnalis $(1 / 84$; $11,1 \%)$ e Hardjoprajitno $(1 / 84 ; 11,1 \%)$. As variáveis quantitativas obtidas através do questionário epidemiológico estão descritas na Tabela 1, enquanto as variáveis qualitativas na Tabela 2. Não houve associação entre a titulação no teste de MAT e as informações obtidas através do questionário epidemiológico, relacionadas ao indivíduo, manejo e controle sanitário dos equinos $(p>0,05)$.

Tabela 1. Variáveis quantitativas obtidas através do questionário epidemiológico aplicado aos responsáveis pelos equinos hospedados no Jockey Club de Pelotas, submetidos à avaliação clínica e exame sorológico de leptospirose.

\begin{tabular}{lcc}
\hline \multicolumn{1}{c}{ Variáveis quantitativas } & MAT $<\mathbf{1 : 1 0 0}(\mathbf{n}=\mathbf{7 5})$ & MAT $\geq \mathbf{1 : 1 0 0}(\mathbf{n}=\mathbf{9})$ \\
\hline Idade (anos) & $4,9 \pm 0,3$ & $4,3 \pm 0,6$ \\
Páreos no JCP (2018/2019) & $2,3 \pm 0,4$ & $1,6 \pm 0,9$ \\
Páreos em outras cidades (2018/2019) & $0,6 \pm 0,2$ & $0,2 \pm 0,1$ \\
\hline
\end{tabular}

Dados descritos como Média \pm EPM. Não houve diferença estatística significativa entre animais com e sem titulação para leptospirose no teste de MAT ( $p>0,05)$. Fonte: Autores. 
Tabela 2. Variáveis qualitativas obtidas através do questionário epidemiológico aplicado aos responsáveis pelos equinos hospedados no Jockey Club de Pelotas, submetidos à avaliação clínica e exame sorológico de leptospirose.

\begin{tabular}{|c|c|c|c|c|c|}
\hline & \multirow[t]{2}{*}{ Variáveis qualitativas } & \multicolumn{2}{|c|}{$\begin{array}{c}\text { MAT }<1: 100 \\
(n=75)\end{array}$} & \multicolumn{2}{|c|}{$\begin{array}{c}\text { MAT } \geq 1: 100 \\
(n=9)\end{array}$} \\
\hline & & Frequência & $(\%)$ & Frequência & $(\%)$ \\
\hline \multirow{3}{*}{ Gênero } & Fêmea & 22 & 29,3 & 1 & 11,1 \\
\hline & Macho castrado & 9 & 12 & 1 & 11,1 \\
\hline & Macho inteiro & 44 & 58,7 & 7 & 77,8 \\
\hline \multirow{3}{*}{ Tipo de cama } & Maravalha & 39 & 52 & 7 & 77,8 \\
\hline & Casca de arroz & 29 & 38,7 & 2 & 22,2 \\
\hline & Maravalha + casca de arroz & 7 & 9,3 & 0 & 0 \\
\hline \multirow{5}{*}{ Dieta } & Alfafa & 75 & 100 & 9 & 100 \\
\hline & Ração comercial & 68 & 90,7 & 9 & 100 \\
\hline & Aveia & 74 & 98,7 & 9 & 100 \\
\hline & Milho & 49 & 65,3 & 6 & 66,7 \\
\hline & Sal mineral & 61 & 81,3 & 6 & 66,7 \\
\hline \multirow{4}{*}{$\begin{array}{l}\text { Armazenamento de } \\
\text { grãos }\end{array}$} & Saco & 24 & 32 & 4 & 44,4 \\
\hline & Tonel & 24 & 32 & 1 & 11,1 \\
\hline & Baú & 18 & 24 & 1 & 11,1 \\
\hline & Saco + tonel & 9 & 12 & 3 & 33,3 \\
\hline \multirow{5}{*}{ Outras informações } & Vacinação contra leptospirose & 1 & 1,3 & 0 & 0 \\
\hline & Animais de estimação & 72 & 96 & 9 & 100 \\
\hline & Acesso dos animais à sala da ração & 43 & 57,3 & 8 & 88,9 \\
\hline & Histórico de roedores & 75 & 100 & 9 & 100 \\
\hline & Moradores no local & 51 & 68 & 5 & 55,9 \\
\hline
\end{tabular}

Dados descritos como Frequência e Percentual. Não houve diferença estatística entre animais com e sem titulação para leptospirose no teste de MAT ( $p>0,05)$. Fonte: Autores.

\section{Discussão}

A partir deste estudo verificou-se que a soroprevalência para leptospirose no Jockey Club de Pelotas no ano de 2019 foi baixa, com apenas 10,7\% (9/84) dos equinos sororreagentes para L. interrogans. Dos sorovares identificados, os que se encontraram em maior proporção foram Canicola e Grippotyphosa, os quais estavam presentes em três dos nove animais sororreagentes. Outro estudo recente avaliou a soroprevalência para L. interrogans em equinos de tração na cidade de Pelotas e região e identificou os mesmos sorovares, porém, os mais frequentes foram Canicola, Hadjo, Ballun 4E e Autumnalis (Hack et al., 2018).

São escassos os estudos soroepidemiológicos avaliando a soroprevalência de L. interrogans em cavalos de corrida (Hamound et al., 2011; Hamound et al., 2012; Bolwell et al., 2020). Estudos realizados no Jockey Club Brasileiro, localizado no Rio de Janeiro, demonstraram títulos reativos em 58,3\% (Hamound et al., 2011) e 71,4\% (Hamound et al., 2012) dos animais. Os autores sugerem que o terreno plano propenso a alagamentos pode ter corroborado com o elevado número de animais sororreagentes (Hamound et al., 2012). Apesar da geografia plana da cidade de Pelotas, o que dificulta o escoamento da água nos períodos de aumento dos índices pluviométricos, o mesmo não foi observado no JCP. Além disso, todos os animais com titulação apresentaram reatividade contra o sorovar Copenhageni (Hamound et al., 2011; Hamound et al., 2012), enquanto no presente estudo, apenas um equino apresentou titulação contra este sorotipo. 
Dos animais avaliados nesse estudo, apenas um equino havia sido vacinado contra leptospirose 15 meses antes da coleta de sangue. Contudo, o mesmo não apresentou titulação contra L. interrogans. Apesar da vacinação não ser uma prática comum no local de estudo, outras medidas profiláticas têm sido adotadas pelos responsáveis, como o uso de tonéis ou baús para armazenamento do concentrado fornecido aos equinos, o que diminui o contato do alimento com roedores e outros animais. Além disso, os equinos permanecem grande parte do tempo nas cocheiras, diminuindo o contato entre os animais. Foi relatado entre os entrevistados a prática de tratar preventivamente os equinos com a associação de penicilina e estreptomicina, mesmo sem a realização de exames diagnósticos para determinar se o animal apresenta altas titulações para L. interrogans, indicando uma infecção aguda. Apesar desta prática ser empírica, esta associação é considerada a terapia de eleição para leptospirose equina (Thomasian, 2005) e pode estar relacionada ao bloqueio da indução de anticorpos anti-leptospiras, controle da leptospiúria e, consequentemente, controle da doença no local de estudo (Girio et al., 2005).

A soroprevalência para leptospirose foi baixa nos animais avaliados neste estudo, o que dificultou a identificação de fatores de risco que estejam associados à leptospirose em cavalos de corrida. A aplicação de questionários epidemiológicos em outros hipódromos, com maior número de animais sororreagentes, pode auxiliar na determinação e combate de fatores de risco associados a esta doença de importância para a saúde pública. É importante ressaltar que titulações iguais ou superiores a 1:100 não significam que o equino apresenta um quadro de leptospirose, mas que houve contato com o agente de forma natural ou através da vacinação. Um único resultado positivo possui valor diagnóstico limitando, sendo recomendada a coleta pareada de soro em áreas endêmicas (Donahue \& Williams, 2000).

\section{Conclusão}

Verificou-se que a soroprevalência para leptospirose entre cavalos de corrida no JCP no ano de 2019 foi baixa, com apenas $10,7 \%$ dos equinos sororreagentes para L. interrogans, o que sugere que este local não é uma fonte de contaminação para a cidade de Pelotas/RS.

\section{Agradecimentos}

Agradecemos ao Jockey Club de Pelotas por ter possibilitado a realização deste estudo e aos órgãos de fomento CNPq e CAPES pelas bolsas de estudo concedidas.

\section{Referências}

Bolwell, C. F., Rogers, C. W., Benschop, J., Collins-Emerson, J. M., Adams, B., Scarfe, K. R. \& Gee, E. K. (2020). Seroprevalence of Leptospira in Racehorses and Broodmares in New Zealand. Animals, 10(11), 1952-1970.

Chang, Y. F. (2021). The reproductive syndrome in equine leptospirosis. Equine Veterinary Journal, 53(4), 856.

Dewes, C., Silva, J. P. M., Fortes, T. P., Marmitt, I. V. P., Vasconcellos, F. A., Felix, S. R., \& Silva, E. F. (2020). Prevalence of anti-Leptospira antibodies in cattle confined for exportation. Research, Society and Development, 9(11), e3329119929.

Divers, T. J., Chang Y. F., Irby, N. L., Smith, J. L., \& Carter, C. N. (2019). Leptospirosis: An important infectious disease in North American horses. Equine Veterinary Journal, 51(3), 287-292.

Donahue, J. M. \& Williams, N. M. (2000). Emergent causes of placentitis and abortions. Veterinary Clinics of North America: Equine Practice, 16(3), 443456.

Ebani, V., Bertelloni, F., Pinzauti, P., \& Cerri, D. (2012). Seroprevalence of Leptospira spp. and Borrelia burgdorferi sensu lato in Italian horses. Annals of Agricultural and Environmental Medicine, 19(2), 237-240.

Faine, S. (1999). Leptospira and Leptospirosis. Medical Science.

Girio, T. M. S., Magajevski, E. S., Girio, R. J. S., Miashyro, S., Rodrigues, L. H., Scarcelli, E. P. \& Toma, S. B. (2005). Uso de estreptomicina na eliminação de leptospiúria em touros (Bos Taurus Indicus) naturalmente infectados pelo sorovar Hadjo. Arquivos do Instituto Biológico, 72(2), 161-170. 
Research, Society and Development, v. 10, n. 8, e29910817287, 2021

(CC BY 4.0) | ISSN 2525-3409 | DOI: http://dx.doi.org/10.33448/rsd-v10i8.17287

Hack, J. D., Mousquer, M. A., Reis, A. S., Dewes, C., Souza, R. P., Ferreira, N. O., Bruhn, F. \& Nogueira, C. E. W. (2018). Soroprevalência de Leptospira interrogans e flaviviroses em equinos da cidade de Pelotas e região, no Rio Grande do Sul. Science and Animal Health, 6(3), 228-246.

Hamond, C., Martins, G. \& Lilenbaum, W. (2012). Subclinical leptospirosis may impair athletic performance in racing horses. Tropical Animal Health and Production, 44(8), 1927-1930.

Hamond, C., Martins, G., Reis, J., Kraus, E., Pinna, A. \& Lilenbaum, W. (2011) Pulmonary hemorrhage in horses seropositive to leptospirosis. Pesquisa Veterinária Brasileira, 31(5), 413-415.

Levett, P. N. (2001). Leptospirosis. Clinical Microbiology Reviews, 14(1), 296-326.

Miraglia, F., Matsuo, M., Morais, Z. M., Dellagostin, O. A., Seixas, F. K., Freitas, J. C., Hartskeerl, R., Moreno, L. Z., Costa, B. L., Souza, G. O., Vasconcellos, S. A. \& Moreno, A. M. (2013). Molecular characterization, serotyping, and antibiotic susceptibility profile of Leptospira interrogans serovar Copenhageni isolates from Brazil. Diagnostic Microbiology and Infectious Disease, 77(3), 195-199.

Santa Rosa, C. A. (1970). Diagnóstico laboratorial das leptospiroses. Revista Microbiológica, 1(2), 97-109.

Sellon, D. C. \& Long, M. T. (2007). Equine infectious diseases. Elsevier.

Thomassian, A. (2005). Enfermidades dos Cavalos. Varela Editora e Livraria.

Verma, A., Stevenson, B. \& Adler, B. (2013). Leptospirosis in horses. Veterinary Microbiology, 167(12), 61-66.

World Health Organization. (2003). Human Leptospirosis. Guidance for Diagnosis, Surveillance and Control. 